\title{
The design of computer controlled ground information transmission down platform in drilling
}

\author{
Dongqiu Xing ${ }^{1, *}$, Lihua $\mathrm{Qi}^{1}$, Jing Zhao ${ }^{1}$ and Yi Wang $^{2}$ \\ ${ }^{1}$ Information Communications Academy, National University of Defense Technology, No.5 \\ Guangming Road, Wangqu Town, Chang'an District, Xi'an City, 710106, China \\ ${ }^{2}$ Xi'an Institute of Applied Optics, No.9 Dianzisan Road, Dianzicheng Town, Yanta District, Xi'an \\ City, 710102, China
}

Keywords: Rotation-guided closed-loop drilling, the down link command, Experimental platform.

\begin{abstract}
In order to improve the drilling efficiency and avoid the disadvantages of manual operation, the method of sending the surface intervention instruction with computer instruction is researched. Based on the similarity of liquid flow, a set of hydraulic circulation system is used to simulate the drilling process, selected the key components, designed and built the intelligent drilling system indoor experiment platform; Define different commands with the length of the time and decode them and carried out the sending command experiment. The results show that the platform can successfully send the command to the downhole under the condition of non-stop drilling, which is the attitude intervention, command coding and Weak signal detection in the intelligent drilling system provides a new platform.
\end{abstract}

\section{Introduction}

An advanced drilling technology in the world includes Rotation-guided closed-loop drilling technology [1]. It is an intelligent closed-loop system. In order to make the well trajectory meet the preset requirements, the surface workers must adjust the attitude of the downhole drilling tools [2]. In the technology that underground to surface transmission of information, there have been mature products that transmit information through mud pulse [3].In terms of surface to downhole information transmission methods, foreign research mainly focuses on the use of electromagnetic wave and mud for signal transmission[4], while domestic research mainly uses mud pulse combination and drill pipe drilling rate change . These technologies have their own advantages and disadvantages, so they are all in the principle scheme stage. in order to reduce cost and as far as possible use of mature technology, in recent years, domestic studies have focused on mud transmission scheme [6], at present this

\footnotetext{
*Corresponding author: Angelaxing@126.com
} 
technology has not been a breakthrough, downward drilling to achieve control instruction still need interrupt drilling, Obviously, this will greatly reduce drilling efficiency, so it is imperative to study a method of downlink instruction without stopping the drilling.

\section{Model establishment}

Normally, the mud flow in the drilling platform is provided by the mud pump in the surface mud pit. The composition of the actual drilling equipment is shown in FIG. 1. The mud enters the drill pipe along the direction of the arrow in the riser, reaches the drill string, passes through the bit nozzle, and finally returns to the surface mud pit from the annulus cavity of the borehole to form the circulation system. At this point, the pressure sensor in the drill bit senses the pressure of the slurry. The branch pipe is introduced out from the riser, and the branch pipe is connected to the switch valve. When the switch valve is opened, the mud flows instantaneously, causing the pressure in the riser and the drill bit to plummet. When the switch valve is closed, the pressure of the circulation system gradually returns to normal. A series of negative mud pulses are generated between the opening and closing of the switch valve. When the switch valve is opened, the instantaneous acceleration of mud flow in the branch pipeline is large, which may lead to pressure rebound. In order to avoid such a situation, it is necessary to connect the switch valve with the mud pool with a pipe with a larger diameter, and increase the accumulator to stabilize the liquid flow. In addition, in order to control the liquid flow pressure, a set of throttle valves can be added on this basis. In order to protect the switch valve and prevent mud from flowing back from the pipe, a one-way valve must also be installed on the pipe. Manual valves can also be installed on the tubing as necessary to ensure the reliability and safety of the drilling system.

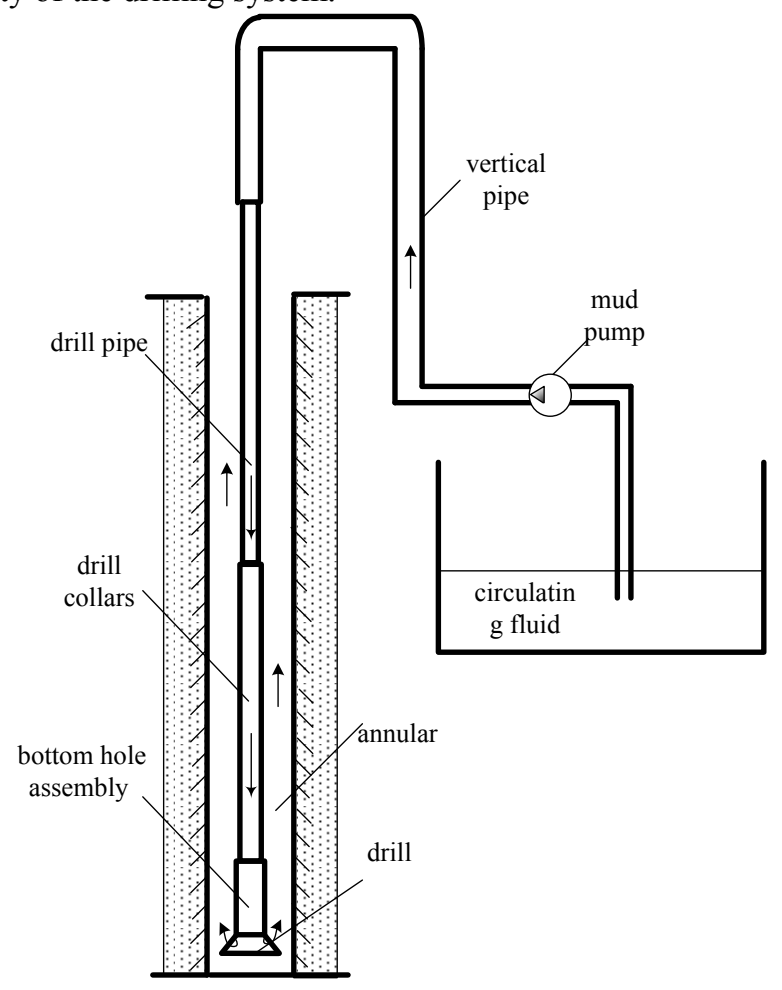

Fig. 1. Actual drilling equipment composition. 


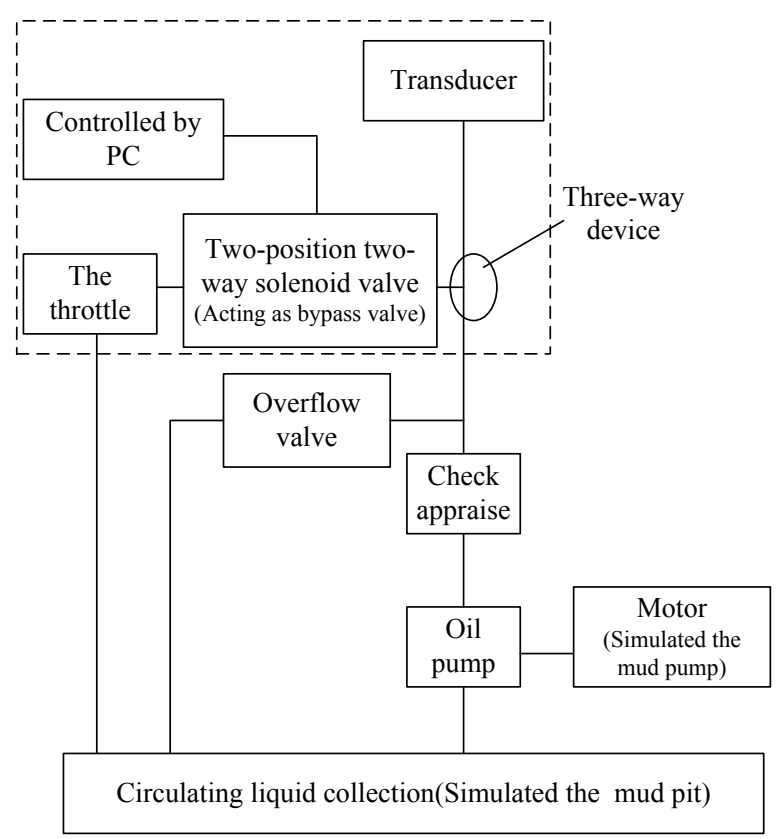

Fig. 2. Schematic diagram of a simulated drilling equipment.

A set of hydraulic circulation system is designed to simulate the actual drilling cycle, as shown in FIG. 2. The bypass valve branch and sensor sensitive device are added in the main circulation loop to meet the requirements of the real device. The three-way pipe with matching diameter integrates the bypass branch, downhole sensitive mechanism and main circulation path, as shown in the dotted line in FIG. 2. The control circuit is designed to open or close the two-position two-way solenoid valve. The solenoid valve is installed in the front end of the sensor equipment. The specific working process of the system is as follows: Start the motor to provide power for the circulation system, the oil pump starts to work, adjust the overflow valve to ensure the highest pressure of the system, when the system pressure is greater than the limit pressure, the high-pressure oil flows back to the collection pool through the overflow valve. Normally the solenoid valve closed, PC control valve body opened to complete the bypass principle. The throttle valve is used to adjust the flow of liquid into the well.In the actual drilling, the distance from the wellhead to the bottom of the well is relatively long, and there will be time delay in the change of system pressure. Therefore, this phenomenon can be simulated by lengthening the tubing line in the experiment.

\section{The construction of the experimental platform for information transmission}

\subsection{Solenoid valve selection}

The displacement of the hydraulic pump cannot be founded. The reference figure for calculation is shown in FIG. 3, where the motor power is used as the premise to calculate:

$$
p_{0}=\frac{p Q}{60}[7]
$$




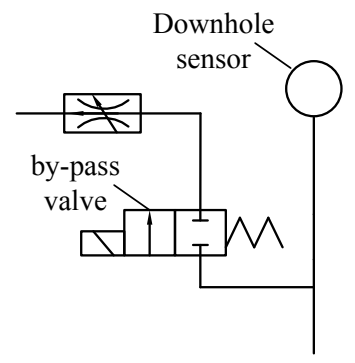

Fig. 3. Schematic diagram of bypass calculation.

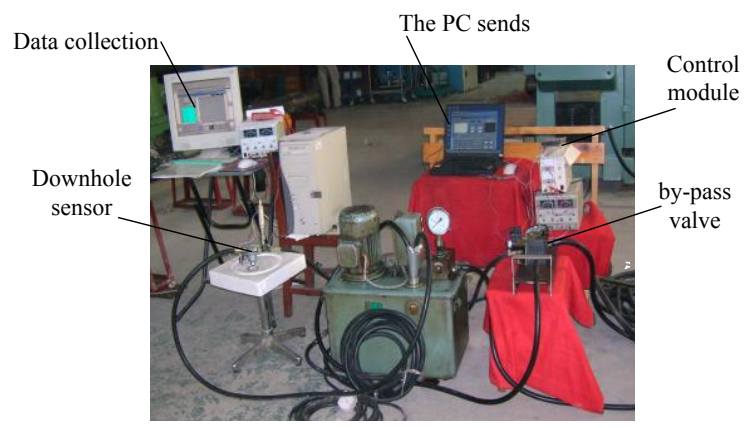

Fig. 4. Experimental platform equipment.

( $\mathrm{p}_{0--}$ motor power $(\mathrm{KW}) ; \mathrm{p}---$ the working pressure of the system (4MPa); $\mathrm{Q}$ - --flow $(\mathrm{l} / \mathrm{min})$

After calculation, $\mathrm{Q}=16.5 \mathrm{l} / \mathrm{min}$, At present, the common sizes of solenoid valves on the market are $6 \mathrm{~mm}$ and $10 \mathrm{~mm}$, and the minimum sizes of throttle valves are $10 \mathrm{~mm}$. Therefore, three kinds of data are used to calculate the pressure difference of the system. Switch solenoid valves are selected respectively with a diameter of $10 \mathrm{~mm}$ and a flow rate of $401 / \mathrm{min} ; 6 \mathrm{~mm}$, flow rate 10 1/min;

The throttle valve size is $10 \mathrm{~mm}$, and the throttle range is $1.6 \mathrm{l} / \mathrm{min} \sim 40 \mathrm{l} / \mathrm{min}$ for flow differential pressure estimation. According to the formula

$$
\begin{gathered}
\mathrm{q}=\mathrm{C}_{\mathrm{d}} \mathrm{A}_{0} \sqrt{\frac{2 \Delta \mathrm{P}}{\sigma}}[7] \\
\Delta \mathrm{P}=\frac{1}{2}\left(\frac{\mathrm{q}}{\mathrm{C}_{\mathrm{d}} \mathrm{A}_{0}}\right)^{2} \sigma
\end{gathered}
$$

(q-- flow coefficient; Cd-- the cross;A0-sectional area of the flow orifice; $\Delta \mathrm{p}$-- pressure difference before and after closure; $\sigma$--liquid density)

According to the above analysis and calculation, the following conclusions can be drawn: (1) the solenoid valve has a diameter of $10 \mathrm{~mm}$ with a flow rate of $40 \mathrm{l} / \mathrm{min}$ and the throttle valve is all open with a flow rate of $40 \mathrm{l} / \mathrm{min}$. However, the pump flow rate is $16.5 \mathrm{l} / \mathrm{min}$. Therefore, after the solenoid valve is opened, the flow rate at the throttle valve is $16.5 \mathrm{l} / \mathrm{min}$, and the pressure difference at both ends of the throttle valve is 
$\Delta \mathrm{p}=0.008 \mathrm{MPa},(2)$ the size of the solenoid valve $6 \mathrm{~mm}$, the flow of 10 liters/minute, throttle valve is all open state flow every minute 40 liters, so when the solenoid valve open, the system will be at the bottom of the solenoid valve circuit there is high pressure (because the solenoid valve flow is less than the system flow), throttle valve open position without adjustment effect, calculate the pressure difference at both ends of the solenoid valve $\Delta \mathrm{p}=$ $0.024 \mathrm{MPa}$, (3) when the flow at the throttle is $1.61 / \mathrm{min}$, estimate the pressure difference between the two ends of the valve

$\Delta \mathrm{p}=31.3 \mathrm{MPa}$

But at this time, the pressure of the overflow valve control system is maintained at $4 \mathrm{MPa}$.

\subsection{Selection of pressure sensor}

Select pressure sensor the model produced by Beijing avic electromechanical research institute is cyb-20s thin film pressure transmitter, with a range of $0 \sim 40 \mathrm{MP}$ and a power supply requirement of $+5 \mathrm{VDC}$.

\subsection{Selection of pressure sensor}

According to the above theoretical calculation and the selection of solenoid valve and pressure sensor, the established experimental platform is shown in FIG. 4.

\subsection{Pressure sensor calibration}

With $1 \mathrm{MPa}$ as the unit, change the pressure output of the standard manometer $(0 \sim 25 \mathrm{MPa})$, increase and decrease the pressure successively, repeat three times, use the data acquisition circuit to collect the sensitive signal output of the sensor, and obtain the output characteristic curve of the pressure sensor through the measured data as shown in FIG. 5.It is not difficult to see that the output of the sensor is a standard voltage signal with good linearity, which is connected to the signal acquisition circuit board for acquisition.

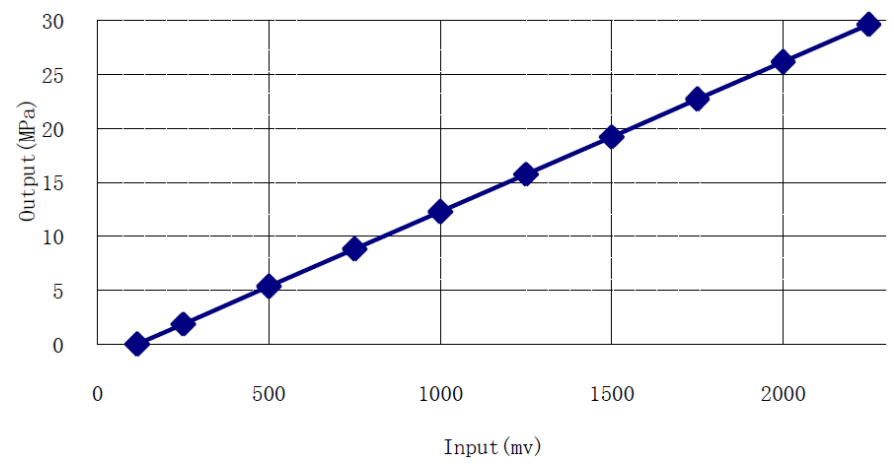

Fig. 5. Sensor output characteristic curve.

\subsection{Control design and valve opening test}

Whether the solenoid valve can be opened as scheduled is the key to send down command by computer control The DC-DC controllable power supply produced by xi 'an spectrum power supply co., ltd. is selected to convert the DC voltage of $+12 \mathrm{~V}$ into the DC voltage of $+24 \mathrm{~V}$. 
When the control signal comes, the output voltage of $24 \mathrm{~V}$ will be available. This design is conducive to reducing the power consumption of the whole system. Choose to conform to the requirements of the equipment 22EH - H6B bidirectional solenoid valve, power consumption of $40 \mathrm{~W}$ or less, through the computer serial port to send command, SCM according to the state, output level by 74LS04 drive circuit power module, main chip P5.0 C8051F060 port output high/low level, according to certain rules, transform the $+12 \mathrm{~V}$ power supply solenoid valve core and the voltage of $+24 \mathrm{~V}$, The electromagnetic valve opens, the bypass oil circuit is conducted, and the pressure in the main circulation system is reduced and monitored by the pressure sensor. The experiment shows that the solenoid valve can work normally, and a group of valve opening data is selected as shown in FIG. 6.

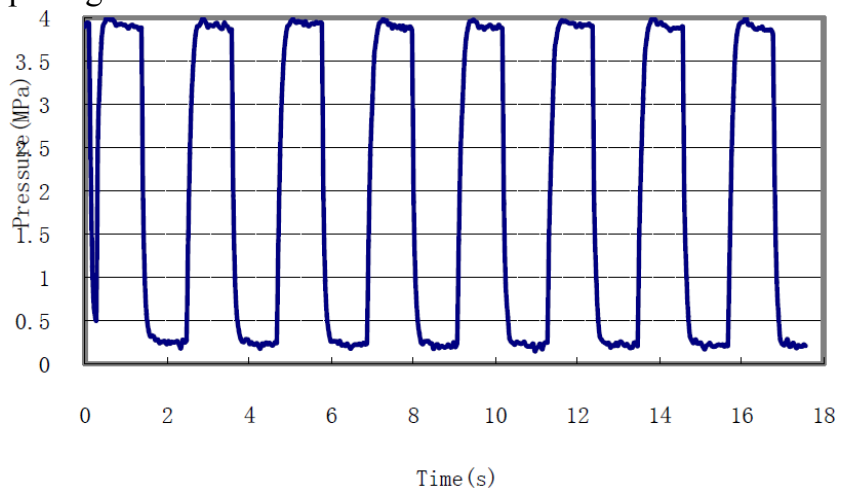

Fig. 6. S Electromagnetic valve working process system pressure change curve.

\subsection{Information transmission experiment controlled by computer}

Block diagram of computer control principle was shown in Fig.7. According to the pre agreed table, the single-chip microcomputer corresponds to a binary data of different values, queries the table and sends out control signals according to the specified time to control the switch of the solenoid valve; when receiving signals in the underground, the corresponding valve status and time interval are converted into binary corresponding decimal command code, so as to recover the command value of the information transmitted from the ground, and the computer controls the transmission The single instruction and the continuous instruction are used to observe the downhole decoding experiment. Select the decoding curve as shown in Fig. 8 the decoding curve of the continuous downlink information instruction. The experimental results show that the software can decode the information instructions correctly by computer .

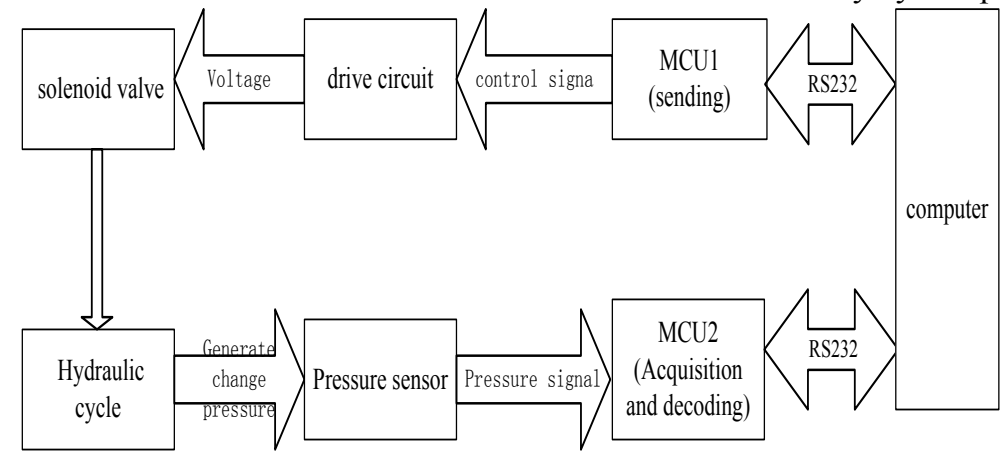

Fig. 7. Block diagram of computer control principle. 


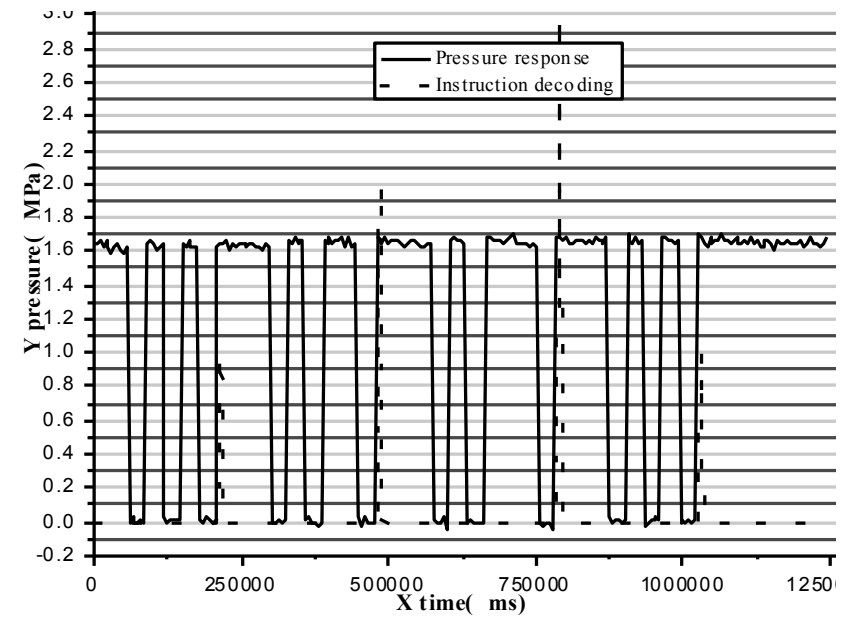

Fig. 8. Decoding curve of the downlink information instruction.

\section{Conclusion}

Based on the theory of intelligent drilling system, and built a set of indoor simulation experiment platform for attitude intervention of downhole drilling tools, selected the key components in the experiment platform, and explained the principle and function of the main components in detail. Designed coding method, correct decoding verified the feasibility of design coding method. The experimental data show that the experimental platform can realize the downhole instruction transmission under the computer control during drilling, which provides the reference for the research on the information engineering under the computer control. At the same time, it can also provide experimental verification platform for coding method research, downhole complex environment construction and weak signal detection.

\section{References}

1. Wang Yeqiang, Research on Guiding Force Distribution of Rotary Steering Drilling Tool.( 2016) 2-3.

2. Chen hongxin, Dang ruirong, Jiang shiquan ect., Pressure testing under command in drilling operations device. China Patent:CN201420548.(2013)

3. Zhang Xiliang, Ma Renqi Research and Application of Contactless Electrical Energy Transmission Technology for Downhole Petroleum Instrument,Petroleum Tubular Goods \& Instruments April.(2017)45-47.

4. Chengdu Hu, He Pen-fei etc.. Application of China made Rotary Steering and Logging while Drilling Systems in Adjustment Well of Bohai,Exploration Engineering (Rock \& Soil Drilling and Tunneling), Mar. (2017)35-38.

5. Xue Qilong, Ding Qingshan etc., The Latest Progress and Development Trend of Rotary Steering Drilling, Technology China Petroleum Machinery, Jan(2013)1-7.

6. Liu Pengfei, HE Pengfei, LI Fan, YUAN Hongshui,PENG Jiang, Open-hole sidetrack drilling technique for $\mathrm{C} 33 \mathrm{H}$ under-displacement horizontal well, Oil Drilling \& Production Technology. 36(1) (2014)44-47.

7. Modern comprehensive mechanical design manual (part 2) Beijing, Beijing press,(2000). 